CERN-TH/98-48

UCLA/98/TEP/4

February 1998

\title{
Horizon Geometry, Duality and Fixed scalars in Six Dimensions"
}

\author{
L. Andrianopoli ${ }^{a, c}$, R. D'Auria ${ }^{b}$, S. Ferrara ${ }^{c}$, M.A. Lledód,e. \\ ${ }^{a}$ Dipartimento di Fisica, Università di Genova, via Dodecaneso 33, I-16146 \\ Genova and Istituto Nazionale di Fisica Nucleare (INFN)-Sezione di Torino, \\ Italy. \\ ${ }^{b}$ Dipartimento di Fisica, Politecnico di Torino, Corso Duca degli Abruzzi, \\ 24, I-10129 Torino and Istituto Nazionale di Fisica Nucleare (INFN)-Sezione \\ di Torino, Italy. \\ ${ }^{c}$ CERN Theoretical Division, CH 1211 Geneva 23, Switzerland. \\ ${ }^{d}$ Centro de Física. Instituto Venezolano de Investigaciones Científicas \\ (IVIC). Apdo 21827 Caracas 1020-A. Venezuela. \\ e Physics Department, University of California, Los Angeles. 405 Hilgard \\ Av. Los Angeles, CA 90095-154\%. USA.
}

\begin{abstract}
We consider the problem of extremizing the tension for BPS strings in $D=6$ supergravities with different number of supersymmetries. General formulae for fixed scalars and a discussion of degenerate directions is given. Quantized moduli, according to recent analysis, are supposed to be related to conformal field theories which are the boundary of three dimensional anti-de Sitter space time.
\end{abstract}

${ }^{*}$ Work supported in part by EEC under TMR contract ERBFMRX-CT96-0045 (LNF Frascati, Politecnico di Torino and Univ. Genova), Angelo Della Riccia fellowship, CONICIT fellowship and by DOE grant DE-FG03-91ER40662 


\section{Introduction}

Recently possible connections of brane dynamics of the world volume theory and supergravity in the ambient space of near horizon geometry have been proposed and investigated [1, 6, 3, 4, 7, 6, 2]. These attempts heavily rely on the correspondence of supergravity on an anti-de Sitter bulk and special properties of "topological" singleton representations confined on anti-de Sitter boundaries [8].

Interestingly enough, as pointed out by Maldacena [1], this correspondence can only occur at some special points of the moduli space of the theory. Indeed this observation is based on previous recent analysis which shows that certain moduli fields satisfy, in a given black brane background, flow equations [9] with an attractor point at the horizon where the geometry becomes anti-de Sitter [11.

Fixed scalars (attractors) have been until recently mainly investigated in connection to black holes in $D=4$ and black holes and black strings in $D=5$.

In $D=5$ strings are dual (magnetic) to black holes so fixed scalars in these cases are easily obtained for both strings and black holes by using dual formulae replacing the BPS mass of a black hole with the BPS tension of a string [28]. However, the recent developments on possible dualities between anti-de Sitter spacetimes and brane dynamics has led to focus on string horizon geometries at $D=6$ because of their relation to two dimensional conformal field theories [1, 3, 5, 29].

In the present paper we generalize the analysis of fixed scalars to $D=$ 6 BPS strings, a case which has not been thoroughly treated in previous investigations. We will examine cases with 8,16 and 32 supersymmetries and explicitely compute fixed scalars for all these cases. A discussion of particular theories to which these results apply will also be given.

\section{Central charges and BPS tension in $D=6$ supergravity theories}

We recall here some properties of the moduli dependence of BPS string tension in six dimensional theories.

At $D=6,3$-form field strengths can be self-dual or antiself-dual. Let us denote by $P, Q$ the number of self-dual and antiself-dual field strengths 
respectively.

For all $D=6$ theories we can use a unified formalism based on an underlying moduli space relevant to the problem, locally of the form $\mathrm{O}(P, Q) / \mathrm{O}(P) \times$ $\mathrm{O}(Q)$, where the range of $P, Q$ will depend on the theory [22, 23]. More specifically for theories with $(1,0)$ supersymmetry $P=1, Q=n$. For $(1,1)$ supersymmetry $P=Q=1$, for $(2,0)$ supersymmetry $P=5, Q=n$ and for $(2,2)$ theories $P=Q=5$. Moreover, since $(1,0)$ and $(2,0)$ theories are chiral, $n$ is constrained by the anomaly cancellation to be $n=21$ in $(2,0)$ theories and $n=\left(273+n_{V}-n_{H}\right) / 29$ in $(1,0)$ theory $\left(n_{H}, n_{V}\right.$ being the number of hyper and vector multiplets respectively)?.

The coset space $\mathrm{O}(P, Q) / \mathrm{O}(P) \times \mathrm{O}(Q)$ can be defined by coset representatives $\left.X_{r \Lambda}, X_{I \Lambda}, r=1, \ldots P, I=1, \ldots Q, \Lambda=1, \ldots P+Q\right)$ satisfying the conditions

$$
X_{r \Lambda} X_{r \Sigma}-X_{I \Lambda} X_{I \Sigma}=\eta_{\Lambda \Sigma}
$$

Explicitly, $X_{r I}$ are $P Q$ coordinates and:

$$
X_{r s}=\sqrt{\delta_{r s}+X_{r I} X_{s I}} ; \quad X_{I J}=\sqrt{\delta_{I J}+X_{r I} X_{r J}} .
$$

To define BPS string tension through the BPS condition, we must introduce a "metric" $\mathcal{N}_{\Lambda \Sigma}$ for the self-dual and antiself-dual 3-forms $H^{\Lambda}$. The metric $\mathcal{N}_{\Lambda \Sigma}$ is defined as follows [12]

$$
\mathcal{N}_{\Lambda \Sigma}=X_{r \Lambda} X_{r \Sigma}+X_{I \Lambda} X_{I \Sigma}=2 X_{r \Lambda} X_{r \Sigma}-\eta_{\Lambda \Sigma}
$$

with the property:

$$
\mathcal{N} \eta \mathcal{N} \eta=\mathbb{1}, \quad \text { i.e. } \quad \mathcal{N}^{-1}=\eta \mathcal{N} \eta
$$

The self-duality condition of the field strengths becomes:

$$
\mathcal{N}_{\Lambda \Sigma}{ }^{*} H^{\Sigma}=\eta_{\Lambda \Sigma} H^{\Sigma}
$$

which therefore implies, by integrating Eq. 5 on a 3-sphere

$$
q_{\Lambda}=\eta_{\Lambda \Sigma} m^{\Sigma}
$$

$q_{\Lambda}, m^{\Lambda}$ being the electric and magnetic charges respectively.

\footnotetext{
${ }^{2}$ The construction of $\mathrm{D}=6$ chiral theories has been recently completed in 24
} 
Therefore, $P$ forms have $q=m$ and $Q$ have $q=-m$ as appropriate for self-dual (antiself-dual) forms. Note that the Dirac-Zwanzinger-Schwinger quantization condition for dyonic odd p-forms is [12] [13]

$$
q_{\Lambda} m^{\Lambda}+m^{\Lambda} q_{\Lambda}^{\prime}=2 \pi k
$$

Using the equation above we get the manifestly $\mathrm{O}(P, Q)$ invariant condition

$$
q_{\Lambda} \eta^{\Lambda \Sigma} q_{\Sigma}^{\prime}=\pi k
$$

We note that chiral theories, with $P \neq Q$, are non-lagrangian because of a net number of self- (antiself-) dual tensors [22], [23]. However for non-chiral theories, with $P=Q$, Eq. 5 can be replaced by $P$ unconstrained 2 -form fields for which an action exists. In an appendix we give the relation between the lagrangian couplings of non-chiral theories and the matrix $\mathcal{N}_{\Lambda \Sigma}$ appearing in Eq. 5.

From the quantized set of charges $q$ we must construct "dressed" charges, appropriate to discuss BPS states. They are defined as follows,

$$
Z_{r}=X_{r}^{\Lambda} q_{\Lambda}, \quad Z_{I}=X_{I}^{\Lambda} q_{\Lambda}
$$

with the property

$$
W=Z_{r} Z_{r}+Z_{I} Z_{I}=q^{\Lambda} \mathcal{N}_{\Lambda \Sigma} q^{\Sigma}, \quad Z_{r} Z_{r}-Z_{I} Z_{I}=q^{\Lambda} \eta_{\Lambda \Sigma} q^{\Sigma}
$$

Note that the first of the above equations defines an analogous of the Weinhold potential [14] for $D=6$, BPS strings. The physical meaning of $Z_{r}$ and $Z_{I}$ is that they provide the 3 -form charge eigenstates which enter in the self-dual tensor of the gravitational sector and the antiselfdual matter tensor of $(1,0)$ and $(2,0)$ theories respectively. For the $(1,1)$ and $(2,2)$ theories $Z_{r}$ and $Z_{I}$ all belong to the gravity multiplet. Let us note that $Z_{r}$ (and not $\left.Z_{I}\right)$ is a "central charge" which occurs in the chiral supersymmetry algebra, while in the non chiral case both $Z_{r}$ and $Z_{I}$ are central charges.

For $(1,0)$ theory we have one $Z$ phile for $(2,0)$ theories $Z_{r}$ is a 5 of USp(4). This means that in both cases there is only one central charge eigenvalue and only one type of BPS state (1/2 BPS).

\footnotetext{
${ }^{3}$ In the $(1,1)$ theory $Z^{2}=Z_{L}^{2}=Z_{R}^{2}+e m$ where $Z_{L}\left(Z_{R}\right)$ is the central charge of the $(1,0)((0,1))$ subalgebra and $e, m$ denote the electric and magnetic charges of the BPS string. Both $1 / 2$ and $1 / 4$ BPS strings exist in this case.
} 
For the $(2,2)$ theory, $Z_{r}$ and $Z_{I}$ are in the 5's of two different $\operatorname{USp}(4)$. Therefore, there are two independent central charge eigenvalues and 1/4 BPS states are those for which $Z_{r}^{2} \neq Z_{I}^{2}$ t while $1 / 2$ BPS correspond to light-like charges $q^{\Lambda} \eta_{\Lambda \Sigma} q^{\Sigma}=0$ 15.

We also remark that the $(1,0)$ geometry of tensor multiplets is entirely analogous to the $\mathrm{D}=5$ "very special" geometry [16] for vector multiplets in respect to the fact that to each tensor multiplet corresponds one real scalar as for vectors in $\mathrm{D}=5$. It is not then surprising that the central charge and the moduli geometry carries a resemblance from that case.

First observe that the potential is now

$$
W=Z^{2}+Z_{I}^{2}, \quad Z=X^{\Lambda} q_{\Lambda}
$$

where

$$
Z_{I}=P_{I}^{i} \partial_{i} Z
$$

and $P_{I}^{i}$ is the inverse Vielbein of $\mathrm{O}(1, n) / \mathrm{O}(n)$. Then it follows that

$$
Z_{I}^{2}=G^{i j} \partial_{i} Z \partial_{j} Z
$$

and in particular the condition $Z_{I}=0$ is equivalent to $\partial_{i} Z=0$.

From the previous equations we also note the relation

$$
G_{i j}=\partial_{i} X^{\Lambda} \mathcal{N}_{\Lambda \Sigma} \partial_{j} X^{\Sigma}, \quad\left(X^{\Lambda} \eta_{\Lambda \Sigma} X^{\Sigma}=1\right)
$$

which follows from the Maurer-Cartan equations:

$$
\partial_{i} X_{\Lambda}=X_{\Lambda I} P_{, i}^{I}
$$

and the relation:

$$
\mathcal{N}^{\Lambda \Sigma} X_{\Sigma I}=X_{I}^{\Lambda}
$$

The explicit expressions of $G_{i j}$ and $\mathcal{N}_{\Lambda \Sigma}$ are

$$
\begin{aligned}
G_{i j} & =\delta_{i j}-\frac{x_{i} x_{j}}{1+x_{i}^{2}}, \quad x_{0}=\sqrt{1+x_{i}^{2}}, \quad x^{i}=x_{i} \\
\mathcal{N}_{\Lambda \Sigma} & =2 X_{\Lambda} X_{\Sigma}-\eta_{\Lambda \Sigma}
\end{aligned}
$$

where Eq. 18 follows from:

$$
\begin{aligned}
X_{\Lambda} X_{\Sigma}-X_{\Lambda I} X_{\Sigma I} & =\eta_{\Lambda \Sigma} \\
X_{\Lambda} X_{\Sigma}+X_{\Lambda I} X_{\Sigma I} & =\mathcal{N}_{\Lambda \Sigma}
\end{aligned}
$$

\footnotetext{
${ }^{4}$ By $Z_{r}^{2}$ we mean $Z_{r} Z_{r}$
} 


\section{$3 \quad$ Fixed scalars and Horizon geometry}

From general considerations based on the relation of brane dynamics to asymptotic horizon geometry we expect [12] that the flow of moduli toward the horizon implies an "attractor" condition equivalent to the extremization of $W$ as defined by Eq. 10 .

This condition signals "supersymmetry enhancement" in the horizon geometry [10, 14]. It is remarkable that such "phase transition" occurs at "special points" of the "asymptotic" moduli space of the theory.

In $\mathrm{D}=4,5$ black holes and strings the asymptotic geometry is $\mathrm{Ad}_{2} \times \mathrm{S}_{2}$ and $\mathrm{Ad}_{3} \times \mathrm{S}_{2}$ respectively [11].

Supersymmetry enhancement of the former, the so called Bertotti-Robinson geometry, is due to the fact that the background is superconformal invariant in a 4-dimensional sense [17]. For $\mathrm{D}=5$ the geometry of the string corresponds to a $(4,0)$ two dimensional conformal field theory with the supersymmetry enhancement in the corresponding superalgebra.

In these theories the "fixed moduli" are the vector multiplets while the hypermultiplets are not fixed by the horizon geometry [9, [10].

In the $\mathrm{D}=6$ case, according to the analysis of Ref. [12], fixed scalars correspond to partners of antiselfdual tensors, i.e. the moduli of $(1,0)$ tensor multiplets. In this case the horizon geometry is $\mathrm{Ad}_{3} \times \mathrm{S}_{3}$.

For theories with higher supersymmetry (i.e. with $\mathrm{P}=5$ ) the degenerate directions should correspond to $\mathrm{D}=6(1,0)$ hypermultiplets, in analogy with the phenomenon in $\mathrm{D}=4,5$ for theories with $N>2$ supersymmetry [18]. Fixed scalars can be obtained following the analysis of Ref. 18] for $\mathrm{D}=4,5$ theories.

The basic point are the Maurer-Cartan equations, satisfied by the one form differentials of the BPS central charges [12].

For a $\mathrm{O}(P, Q) / \mathrm{O}(P) \times \mathrm{O}(Q)$ moduli geometry these equations read

$$
\begin{aligned}
& \nabla Z_{r}=P_{r}^{I} Z_{I} \\
& \nabla Z_{I}=P_{I}^{r} Z_{r}
\end{aligned}
$$

where $P_{r I}$ is the Vielbein one-form $\left(P_{r}^{I}=\delta^{I J} P_{r I}, P_{I}^{r}=\delta^{r s} P_{s I}\right)$. From the first equation we get that the "central charge" $Z_{r}$ is extremized by $Z_{I}=0$, which therefore solves $\delta W=0$. It is important to notice that "fixed scalars" for which $Z_{I}=0$ give a "regular" horizon since in this case the analogous of 
the Bekenstein-Hawking entropy

$$
S=\frac{A^{D-2}}{G_{N}}
$$

takes the value

$$
\left.W\right|_{Z_{I}=0}=q^{\Lambda} \eta_{\Lambda \Sigma} q^{\Sigma}
$$

which therefore requires the charge vector $q$ to satisfy $q^{\Lambda} \eta_{\Lambda \Sigma} q^{\Sigma}=q^{2}>0$ when $P<Q$. For $P=Q$, the solution with $q^{2}<0$ is isomorphic to the previous one by interchanging $Z_{r}$ with $Z_{I}$.

We now consider the details of the general remarks to the four particular theories with 8,16,32 supersymmetries o.

In the $(1,0)$ theory the "attractor equation":

$$
\partial_{i} Z=0
$$

has the unique solution

$$
X_{\Lambda}=\frac{q_{\Lambda}}{\sqrt{q^{2}}}
$$

The string tension at this point is

$$
\left.Z\right|_{e x t r}=\sqrt{q_{\Lambda} q^{\Lambda}}=\sqrt{q_{0}^{2}-q_{i}^{2}}
$$

Note that the hypermultiplet scalars (which must be present in the theory because of anomaly cancellation) do not enter in the discussion so the 3dimensional anti-de Sitter geometry cannot depend on them [8].

For $q^{2}<0$, the equation $\delta W=0$ has a different solution which occurs for tensionless strings, i.e. at the point $Z=0$. This is not an extremum of the string tension, but rather at this point:

$$
\left.W\right|_{Z=0}=\left|Z_{I}\right|^{2}=-q^{2}
$$

A particular solution of this equation is:

$$
X_{i}^{(0)}=\frac{q_{i}}{\sqrt{q_{i}^{2}}} \frac{q_{0}}{\sqrt{q_{i}^{2}-q_{0}^{2}}}
$$

\footnotetext{
${ }^{5}$ The $(1,1)$ theory, having one tensor field, is like the $(1,0)$ theory for $n=1$. However the string is $1 / 4 \mathrm{BPS}$ when it is dyonic [30], while it is $1 / 2 \mathrm{BPS}$ when it is purely electric or magnetic.
} 
Let us now consider the $(2,0)$ theory with $P=5, Q=21$. From the form of $W$ and Eq. 20 we see that

$$
\partial_{i} W=0
$$

occurs at $Z_{I}=0$ if $q^{2}>0$.

Since in this case $i$ takes $5 Q$ values and $Z_{I}=0$ are $Q$ conditions, it means that there are precisely $4 Q$ ( 84 for the case at hand) moduli directions not fixed by Eq. 29. These are precisely the Q hypermultiplets in the $(1,0)$ decomposition of the $(2,0)$ tensor multiplets.

Note that, according to the results of Ref. [19], the relevant solvable algebra decomposition of the coset reads

$$
\operatorname{solv}\left(\frac{\mathrm{O}(5, Q)}{\mathrm{O}(5) \times \mathrm{O}(Q)}\right)=\operatorname{solv}\left(\frac{\mathrm{O}(1, Q)}{\mathrm{O}(Q)}\right)+\operatorname{solv}\left(\frac{\mathrm{O}(4, Q)}{O(4) \times \mathrm{O}(Q)}\right)
$$

where the two factors correspond to the $(1,0)$ tensor- and hyper-multiplets respectively.

Eq. 29 implies that the hypermultiplets do not occur in the solution so $4 Q$ directions are undetermined by the attractor condition and will not occur at the extremum of $W$.

It is straightforward to give a particular solution for the fixed scalars which solves Eq. 29. This is given by

$$
X_{r \Lambda}^{(0)}=\frac{q_{r} q_{\Lambda}}{\sqrt{q_{r}^{2}} \sqrt{q_{r}^{2}-q_{I}^{2}}}
$$

It can be easily shown that at $X_{r I}=X_{r I}^{(0)}$

$$
Z_{r}=\frac{q_{r} \sqrt{q_{r}^{2}-q_{I}^{2}}}{\sqrt{q_{r}^{2}}}
$$

which implies

$$
\left.W\right|_{X=X^{(0)}}=Z_{r}^{2}=q_{r}^{2}-q_{I}^{2}
$$

Note that $X^{(0)}$ is a particular solution of $Z_{I}=0$ since it does not determine the moduli directions $\widehat{X}$ orthogonal to $Z_{r}$

$$
\widehat{X}_{r I}=X_{r I}-\frac{Z_{r} X_{s I} Z_{s}}{Z_{s}^{2}}
$$


Using Eq. 18 it is also possible to give a general formula for the $\mathcal{N}_{\Lambda \Sigma}$ metric at the attractor point. It reads

$$
\left.\mathcal{N}_{\Lambda \Sigma}\right|_{X=X^{(0)}}=\frac{2 q_{\Lambda} q_{\Sigma}}{q^{2}}-\eta_{\Lambda \Sigma}
$$

which is of course consistent with Eq. 33 .

Let us now consider the case $q^{2}<0$. Similar to the $(1,0)$ case, $\delta W=0$ occurs in this case at $Z_{r}=0$ at which point:

$$
\left.W\right|_{Z_{r}=0}=Z_{I}^{2}=-q^{2}
$$

This equation implies that tensionless strings are not extrema of the BPS tension. A particular solution of the moduli at this point is:

$$
X_{r I}^{(0)}=\frac{q_{I}}{\sqrt{q_{I}^{2}}} \frac{q_{r}}{\sqrt{q_{I}^{2}-q_{r}^{2}}}
$$

This solution leaves $5(Q-1)=100$ directions undetermined as it appears from the moduli directions orthogonal to $Z_{I}$ :

$$
\widehat{X}_{r I}=X_{r I}-\frac{Z_{I} X_{r J} Z_{J}}{Z_{J}^{2}}
$$

We finally come to the $(2,2)$ theory with R-symmetry given by $\mathrm{O}(5) \times$ $\mathrm{O}(5) \sim \mathrm{USp}(4)_{L} \times \mathrm{USp}(4)_{R}$. In this case the moduli space is locally $\mathrm{O}(5,5) / \mathrm{O}(5) \times$ $\mathrm{O}(5)$ and the attractor condition

$$
Z_{I}=0
$$

implies that the lower eigenvalue of the central charge matrix vanishes, as in the $D=4,5$ cases [10, 18]. For charges $q^{\Lambda} q_{\Lambda}>0, Z_{r}^{2}>Z_{I}^{2}$, and the "attractor point" gives a $1 / 4$ BPS state with Weinhold potential given by

$$
\begin{array}{ccc}
\left.W\right|_{Z_{I}=0}=q^{2} & \text { if } & q^{2}>0 \\
\left.W\right|_{Z_{r}=0}=-q^{2} & \text { if } & q^{2}<0
\end{array}
$$

This situation corresponds to the BPS orbit $\mathrm{O}(5,5) / \mathrm{O}(4,5)$ according to the analysis of Ref. [15, 20].

One half BPS strings require, on the other hand, two coinciding eigenvalues for the central charge and this requires a light-like orbit $q^{\Lambda} q_{\Lambda}=0$. The 
BPS orbit is in this case $\mathrm{O}(5,5) / \mathrm{IO}(4,4)$ and implies (for all values of the moduli) the identity

$$
Z_{r}^{2}=Z_{I}^{2}
$$

Note that in the previous cases of chiral theories the $1 / 2$ BPS orbits fall into three categories, i.e. $\mathrm{O}(P, Q) / \mathrm{O}(P-1) \times \mathrm{O}(Q)$ for $q^{2}>0, \mathrm{O}(P, Q) / \mathrm{IO}(P-$ $1, Q-1)$ for $q^{2}=0$ and $\mathrm{O}(P, Q) / \mathrm{O}(P) \times \mathrm{O}(Q-1)$ for $q^{2}<0$. Light-like orbits do not correspond to supersymmetry enhancement in this case. This is similar to 1/8 BPS orbits with vanishing entropy for $\mathrm{D}=4$ black holes [15, 21]. Space-like orbits $\left(q^{2}<0\right)$ correspond to the occurrence of tensionless strings [27. The moduli at which the string becomes tensionless do not correspond to an extremum of the string tension.

\section{Concluding remarks}

In this paper we have considered in some details BPS strings in different theories at $D=6$ and studied the extremization of the string tension in the moduli space.

Fixed scalars correspond to particular quantized values in terms of the string charges.

In $(1,0)$ theories the fixed scalars are in tensor multiplets, in $(1,1)$ theory the fixed scalar is in the gravity multiplet (dilaton) and in $(2,0),(2,2)$ theories the fixed scalars correspond to the tensor multiplet scalars resulting by decomposing the theories in $(1,0)$ representations. The other scalar fields are not fixed by the horizon geometry, therefore 84 and 20 moduli are at their initial value in the $(2,0)$ and the $(2,2)$ theories respectively.

The four kinds of $6 D$ supergravity theories considered here correspond, in string theory language, to different compactifications of heterotic and Type II strings on $\mathrm{K}_{3}$ and $\mathrm{T}_{4}$. (1,0) theories with more than one tensor multiplet naturally arise in open string constructions [26] and F-theory compactifications [25]. The fixed scalars correspond to special isolated points in the $K_{3}$ and $\mathrm{T}_{4}$ moduli spaces. These points should play an important role because it is precisely at these values that the horizon geometry $\mathrm{Ad}_{3} \times \mathrm{S}_{3}$ is defined.

3 -d anti-de Sitter supergravity has been recently reconsidered on the new light of possible duality relations between anti-de Sitter physics and the brane (string in this case) conformal dynamics [3, 5, 29]. 


\section{Acknowledgements}

One of us (S.F.) would like to thank J. Maldacena and K. Sfetsos for useful discussions.

\section{A Appendix}

In this appendix we consider the relation of the "coupling" $\mathcal{N}_{\Lambda \Sigma}$ in Eq. 5 and the lagrangian of $P$ unconstrained 2 -form potentials.

The basic lagrangian is:

$$
\frac{1}{2} g_{\Lambda \Sigma} H^{\Lambda} \wedge^{*} H^{\Sigma}+\frac{1}{2} b_{\Lambda \Sigma} H^{\Lambda} \wedge H^{\Sigma}
$$

where $g_{\Lambda \Sigma}$ is the real symmetric kinetic coupling and $b_{\Lambda \Sigma}$ is the real antisymmetric "axionic" coupling.

Decomposing :

$$
H^{\Lambda}=H^{+\Lambda}+H^{-\Lambda}
$$

in self-dual and antiself-dual parts we get:

$$
\frac{1}{2} \mathcal{N}_{+\Lambda \Sigma} H^{+\Lambda} \wedge H^{-\Sigma}+\frac{1}{2} \mathcal{N}_{-\Lambda \Sigma} H^{-\Lambda} \wedge H^{+\Sigma}
$$

with:

$$
\mathcal{N}_{ \pm \Lambda \Sigma}=\mp g_{\Lambda \Sigma}+b_{\Lambda \Sigma}
$$

and therefore:

$$
\mathcal{N}_{+}=-\mathcal{N}_{-}^{T}
$$

$\mathcal{N}_{ \pm}$transforms with a fractional transformation under the $O(P, P)$ action, i.e.:

$$
\mathcal{N}_{ \pm}^{\prime}=\left(C+D \mathcal{N}_{ \pm}\right)\left(A+B \mathcal{N}_{ \pm}\right)^{-1}
$$

where:

$$
\begin{gathered}
\left(\begin{array}{cc}
A & B \\
C & D
\end{array}\right) \in O(P, P): \\
A^{T} C+C^{T} A=B^{T} D+D^{T} B=0 \quad, \quad A^{T} D+C^{T} B=1
\end{gathered}
$$


The relation between $\mathcal{N}_{ \pm}$as given by Eq. 46 and the matrix $\mathcal{N}$ as given in Eq. 5 turns out to be:

$$
\begin{aligned}
\mathcal{M} \equiv C^{T} \mathcal{N} C & =\left(\begin{array}{cc}
2\left(\mathcal{N}_{-}^{-1}-\mathcal{N}_{+}^{-1}\right)^{-1} & \left(\mathcal{N}_{-}+\mathcal{N}_{+}\right)\left(\mathcal{N}_{-}-\mathcal{N}_{+}\right)^{-1} \\
-\left(\mathcal{N}_{-}-\mathcal{N}_{+}\right)^{-1}\left(\mathcal{N}_{-}+\mathcal{N}_{+}\right) & 2\left(\mathcal{N}_{-}-\mathcal{N}_{+}\right)^{-1}
\end{array}\right) \\
& =\left(\begin{array}{cc}
g-b g^{-1} b & b g^{-1} \\
-g^{-1} b & g^{-1}
\end{array}\right)=\left(\begin{array}{ll}
1 & b \\
0 & 1
\end{array}\right)\left(\begin{array}{cc}
g & 0 \\
0 & g^{-1}
\end{array}\right)\left(\begin{array}{cc}
1 & 0 \\
-b & 1
\end{array}\right)
\end{aligned}
$$

where:

$$
C=\frac{1}{\sqrt{2}}\left(\begin{array}{cc}
1 & 1 \\
-1 & 1
\end{array}\right)
$$

The matrix given by Eq. 50 satisfies the property:

$$
\mathcal{M} \widehat{\eta} \mathcal{M} \widehat{\eta}=\mathbb{1}
$$

with respect to the off-diagonal metric:

$$
\widehat{\eta}=\left(\begin{array}{ll}
0 & 1 \\
1 & 0
\end{array}\right)=C^{T} \eta C
$$

The above equation, Eq. 50, is obtained using the results of ref. 31 for the parametrization of the coset representative $X$ of $O(P, P) /[O(P) \times O(P)]$ as explained in ref. [12.

In other words, the $2 P \times 2 P$ matrix $\mathcal{N}$ transforms as a $O(P, P)$ tensor when $\mathcal{N}_{ \pm}$undergo the projective transformation given by Eq. 48 .

\section{References}

[1] J. M. Maldacena, hep-th/9711200.

[2] N. Itzhaki, J. M. Maldacena, J. Sonnenschein and S. Yankielowicz, hepth/9802042.

[3] K. Sfetsos and K. Skenderis, hep-th/9711138.

[4] S. Hyun, hep-th/9704005

[5] H. Boonstra, B. Peeters and K. Skenderis, hep-th/9801076, Phys. Lett. B411 (1997) 59.

[6] P. Claus, R. Kallosh and A. van Proeyen, hep-th/9711161. 
[7] S. Ferrara and C. Fronsdal, hep-th/9712239, hep-th/9802126.

[8] M. Gunaydin and D. Minic, hep-th/9802047 and references therein.

[9] S. Ferrara, R. Kallosh and A. Strominger, Phys. Rev. D52 (1995) 5412.

[10] S. Ferrara and R. Kallosh, Phys. Rev. D54 (1996) 1514, Phys. Rev.D54 (1996) 1525; A. Chamseddine, S. Ferrara, G. Gibbons and R. Kallosh, Phys. Rev. D55 (1997) 364.

[11] G. Gibbons and P.K. Townsend, Phys. Rev. Lett. 71 (1993) 3754.

[12] L. Andrianopoli, R. D'Auria and S. Ferrara, Int. J. Mod. Phys. A 12 (1997) 3759; hep-th/9612105 to appear in Int. J. Mod. Phys.A.

[13] S. Deser, A. Gomberoff, M. Henneaux and C. Teitelboim, Phys. Lett. B400 (1997) 80; hep-th/9712189, to appear in Nucl. Phys. B; S. Deser, M. Henneaux and A. Schwimmer, "A note on quantization condition for p-brane dyons", to appear.

[14] S. Ferrara, G. Gibbons and R. Kallosh,Nucl. Phys. B500 (1997) 75.

[15] S. Ferrara and J. Maldacena, hep-th/9706097.

[16] M. Gunaydin, G. Sierra and P. Townsend, Nucl. Phys. B242 (1994) 244, Nucl. Phys. B253 (1985) 573; B. de Wit and A. Van Proeyen Phys. Lett. B293 (1992) 94.

[17] R. Kallosh and A. Peet, Phys. Rev. D46 (1992) 5223.

[18] L. Andrianopoli, R. D'Auria and S. Ferrara, Phys. Lett. B403 (1997) 12;Phys. Lett. B411 (1997) 39.

[19] L. Andrianopoli, R. D'Auria and S. Ferrara, P. Fré and M. Trigiante, Nucl. Phys. B496 (1997) 617;L. Andrianopoli, R. D'Auria and S. Ferrara, P. Fré, R. Minasian and M. Trigiante, Nucl. Phys. B493 (1997) 249.

[20] H. Lu, C. Pope and K. Stelle hep-th/9708109.

[21] S. Ferrara, M. Gunaydin hep-th/9708025. 
[22] L. J. Romans Nucl. Phys. B276 (1996) 71.

[23] A. Sagnotti, Phys. Lett. B294 (1992) 196.

[24] S. Ferrara, F. Riccioni and A. Sagnotti hep-th/9711059; F. Riccioni, hep-th/9712176.

[25] C. Vafa, Nucl. Phys. B469 (1996) 403; D. Morrison and C. Vafa Nucl. Phys.B473 (1996) 74, Nucl. Phys. B476 (1996) 437.

[26] A. Sagnotti, Talk at Cargèse Summer Institute (1997), Eds. G. Mack et al. (Pergamon Press (1998) 521; M. Bianchi and A. Sagnotti, Phys. Lett. B247 (1990) 517, Nucl. Phys. B361 (1991) 519.

[27] E. Witten hep-th/9507121; N. Seiberg and E. Witten Nucl. Phys. B471 (1996) 121; M.J. Duff, H. Lu and C.N. Pope, Phys. Lett. B378 (1996) 101.

[28] A. Chou, R. Kallosh, J. Rahmfeld, S.-J. Rey, M. Shmakova and W.K. Wong, Nucl.Phys. B508 (1997) 147.

[29] A. Strominger, hep-th/9712251; J. Maldacena, A. Strominger, grqc/9801096.

[30] M. J. Duff, S. Ferrara, R.R. Khuri and J. Rahmfeld, Phys. lett. B356 (1995) 479.

[31] M.K.Gaillard and B. Zumino, Nucl. Phys. B 193 (1981) 221 